\title{
Overview of Triptans in the Treatment of Acute Migraine
}

\author{
Pietro Cortelli, ${ }^{1,2}$ Gianni Allais ${ }^{3}$ and Chiara Benedetto ${ }^{3}$ \\ 1. Department of Biomedical and NeuroMotor Sciences (DIBINEM), Alma Mater Studiorum - Università di Bologna, Bologna, Italy; \\ 2. IRCCS Istituto delle Scienze Neurologiche di Bologna, Ospedale Bellaria, Bologna, Italy; 3. Women's Headache Center, Department \\ of Surgical Sciences, University of Torin, Turin, Italy
}

DOI: https://doi.org/10.17925/ENR.2017.12.02.71

\begin{abstract}
$\mathrm{T}$ headvent of triptans for effective relief of migraine represented a therapeutic breakthrough. Triptans are serotonin (5-hydroxytryptamine, or 5-HT) agonists with high affinity for 5- $\mathrm{HT}_{1 \mathrm{~B}}$ and 5- $\mathrm{HT}_{1 \mathrm{D}}$ receptors. There are, at present, seven commonly used triptans: almotriptan, eletriptan, frovatriptan, naratriptan, rizatriptan, sumatriptan, and zolmitriptan. Some controversy still surrounds the mode of action of this class. When first studied, it was thought that triptans provided relief from migraine through cranial vasoconstriction, probably via action at postsynaptic 5- $\mathrm{HT}_{1 \mathrm{~B}}$ receptors on the smooth-muscle cells of blood vessels. More recently, however, triptans have also been demonstrated to block release of vasoactive peptides from the perivascular trigeminal neurons owing to their action at presynaptic 5-HT 1 receptors on the nerve terminal. Triptans may also facilitate descending pain inhibitory systems. However, it is not certain whether or not the activation of vascular $5-\mathrm{HT}_{1 \mathrm{~B}}$ receptors is essential for relieving migraine. Many drug characteristics need to be taken into account when selecting the best triptan for an individual patient. Clinical characteristics of the migraine attack and the patient's lifestyle and medical history are also important. Despite their biochemical similarity, triptans have distinct pharmacokinetic and pharmacodynamic profiles. Frovatriptan and naratriptan, for example, have a longer half-life and therefore a delayed onset of action and prolonged duration compared with the other triptans, which are fast acting, with a rapid dose-dependent efficacy and higher risk of adverse events and migraine recurrence. Migraine recurrence is affected by the pharmacological and pharmacokinetic properties of the triptan but is unrelated to initial clinical efficacy. Triptans with a longer half-life and largest $5-\mathrm{HT}_{1 \mathrm{~B}}$ receptor affinity have the lowest rates of headache recurrence.
\end{abstract}

\section{Keywords}

Migraine, triptans, almotriptan, eletriptan, frovatriptan, naratriptan, rizatriptan, sumatriptan, zolmitriptan

Disclosure: Pietro Cortelli has received honoraria for speaking engagements or consulting activities with Allergan Italia, UCB Pharma SPA, Chiesi Farmaceutici, AbbVie SrL, Eli Lilly and Company, and Zambon. Gianni Allais and Chiara Benedetto have no conflicts to disclose in relation to this article.

Acknowledgements: Medical writing assistance was provided by Catherine Amey and Ray Ashton at Touch Medical Media, funded by Menarini.

Compliance with Ethics: This article involves a review of the literature and did not involve any studies with human or animal subjects performed by any of the authors.

Authorship: All named authors meet the International Committee of Medical Journal Editors (ICMJE) criteria for authorship of this manuscript, take responsibility for the integrity of the work as a whole, and have given final approval to the version to be published.

open Access: This article is published under the Creative Commons Attribution Noncommercial License which permits any non-commercial use, distribution, adaptation and reproduction provided the original author(s) and source are given appropriate credit.

Received: 23 February 2017

Accepted: 19 May 2017

Citation: European Neurological Review, 2017;12(2):71-7

Corresponding Author: Gianni Allais,

Women's Headache Center, Department of Surgical Sciences, University of Turin, Via Ventimiglia 3, I-10126, Turin, Italy. E: gb.allais@tiscali.it

Support: The publication of this article was supported by an independent, educational grant provided by Menarini. Menarini had no influence on the development or

content of the article except in reviewing the accuracy of the medical data presented.
Migraine is one of the most common headache disorders causing substantial disability for affected patients and has a considerable societal cost owing to the high prevalence in the general population. ${ }^{1}$ Results from the Global Burden of Diseases, Injuries, and Risk Factors Study 2015 for all-cause mortality, cause-specific mortality and non-fatal disease burden were used to calculate disability-adjusted life-years (DALYS). ${ }^{2}$ Total DALYs and all-age DALY rates for migraine significantly increased by $29.7 \%$ and $5.6 \%$, respectively, between 1990 and 2005 . Using years lived with disability (YLD), migraine is ranked seventh in the world (among the top level-4 causes of disability) for both genders and all age ranges. ${ }^{3}$ In those aged $15-49$ years, migraine is ranked third. The average lifetime prevalence is approximately $18 \%$ and 1 -year prevalence approximately $13 \%{ }^{4-10}$ Women experience migraine around three-times more often than men and prevalence peaks at approximately 45 years of age. ${ }^{11}$

A number of guidelines have been published and recommend the use of pharmacological therapies as first-line treatment for mild-to-moderate migraine in adults. The National Institute for Health and Care Excellence (NICE) published guidelines for England and Wales in 2015.12 These guidelines based on scientific evidence are intended for the primary care setting. At first consultation, the doctor should obtain a complete description and make the diagnosis while excluding serious underlying causes. A triptan should be prescribed plus either a non-steroidal anti-inflammatory drug (NSAID) or paracetamol to treat the attack. Topiramate, propanolol or amitriptyline can be used for prevention, and frovatriptan or zolmitriptan in menstrual migraine. Silberstein et al. assessed pharmacological therapies for the prevention of migraine and identified 29 Class I or Class II articles from published studies. ${ }^{13}$ They concluded that antiepileptic drugs (divalproex sodium, sodium valproate and topiramate), $\beta$-blockers (metoprolol, propanolol and timolol) and triptans (frovatriptan for short-term menstrual migraine prevention) were effective for migraine prevention (Level A - medicines with established efficacy in at least two Class I trials). These drugs should be prescribed to patients with migraine to reduce attack frequency and severity. ${ }^{13}$

Triptans are effective for migraine relief and their use represented a breakthrough in the treatment of migraine. ${ }^{14}$ However, it is unclear which triptan has the greatest efficacy compared with the others in this class and when compared with non-triptan migraine treatments. Cameron et al. assessed 133 randomised, controlled trials (RCTs) of triptans versus placebo or active controls. 
Standard doses of triptans relieved headaches within 2 hours and gave pain relief for 2 hours (42-76\% and $18-50 \%$, respectively). Furthermore, they gave sustained headache relief and freedom from pain. Generally, triptans provided equal or better outcomes than other migraine treatments but slightly worse efficacy than combination therapy. ${ }^{14}$

This review examines the use of the currently licensed triptans for the treatment of acute migraine and practical considerations when selecting a triptan for this indication. The efficacy and safety of the triptans using data from clinical trials and real-world studies will be compared.

\section{Triptans for the treatment of acute migraine}

Triptans are serotonin (5-hydroxytryptamine, or 5-HT) agonists with high affinity for $5-\mathrm{HT}_{1 \mathrm{~B}}$ and $5-\mathrm{HT}_{1 \mathrm{D}}$ receptors. ${ }^{15}$ When the mode of action of triptans was first investigated, it was thought that they gave relief from migraine from their ability to cause cranial vasoconstriction, probably through action at postsynaptic $5-\mathrm{HT}_{1 \mathrm{~B}}$ receptors on the smooth-muscle cells of blood vessels. More recently, it has been discovered that triptans also block the release of vasoactive peptides from the perivascular trigeminal neurons through their action at presynaptic $5-\mathrm{HT}_{1 \mathrm{D}}$ receptors on the nerve terminals. ${ }^{16}$ They also bind to presynaptic $5-\mathrm{HT}_{10}$ receptors in the dorsal horn, which seems to block the release of neurotransmitters that activate second-order neurons ascending to the thalamus. ${ }^{16}$ In addition, they may also facilitate descending pain inhibitory systems. ${ }^{16}$ However, it is not certain whether the activation of vascular $5-\mathrm{HT}_{1 \mathrm{~B}}$ receptors is essential for relieving migraine..$^{15}$

There are currently seven molecules in the triptan class - almotriptan, eletriptan, frovatriptan, naratriptan, rizatriptan, sumatriptan and zolmitriptan. ${ }^{17}$ They are biochemically similar but have distinctive pharmacokinetic and pharmacodynamic properties. For example, naratriptan and frovatriptan have a longer half-life and therefore a delayed onset of action and prolonged duration compared with the other triptans, which are fast acting, with a rapid dose-dependent efficacy and higher risk of adverse events (AEs) and recurrence of the migraine. Thus, many drug characteristics need to be considered when selecting the optimum triptan for an individual patient.

\begin{abstract}
Almotriptan
Almotriptan is a potent, selective $5-\mathrm{HT}_{1 \mathrm{~B}}$ and $5-\mathrm{HT}_{1 \mathrm{D}}$ receptor agonist with approximately $70 \%$ bioavailability when administered orally, which is higher than that for other triptans. ${ }^{18}$ Although the mean half-life is similar to other triptans (3-5 hours), it is significantly lower than frovatriptan (half-life approximately 25 hours). ${ }^{18}$ Almotriptan has been shown to be one of the best-responding triptans for pain relief and pain-free rate at 2 hours with the lowest AES of the triptans. ${ }^{18}$
\end{abstract}

The activity of almotriptan has been evaluated in four controlled, doubleblind, randomised clinical trials. Two were against placebo and two included sumatriptan ( $\mathrm{n}=2,500$ patients with moderate-to-severe episodic migraine). ${ }^{18}$ The first trial assessed almotriptan at 6.25 or $12.5 \mathrm{mg} .{ }^{19}$ Pain relief at 2 hours was significantly higher with almotriptan (60, 70 and $38 \%$ for the $6.25,12.5 \mathrm{mg}$ dose of almotriptan and placebo respectively, $\mathrm{p}<0.001$ ). AEs occurred in $23.1 \%$ of patients, but there were no significant differences between groups. A dose-finding phase II trial assessed a single dose of almotriptan $(2,6.25,12.5$ and $25 \mathrm{mg})$ versus placebo in 742 patients. ${ }^{20}$ The headache response at 2 hours showed a significant dose-dependent increase $(p<0.0001)$, with efficacy being greater than placebo ( $p<0.001$ ) except for the $2 \mathrm{mg}$ dose. In total, $18.7 \%$ of patients had AEs with the incidence and intensity dose-dependent and they were significantly higher at the $25 \mathrm{mg}$ dose. In contrast AEs were similar to placebo for the other doses. These findings indicated that the optimum dose for almotriptan was $12.5 \mathrm{mg}$.

Almotriptan (12.5, $25 \mathrm{mg}$ ) was compared to sumatriptan (100 mg) in a randomised, single-dose, double-blind, parallel-group, placebocontrolled, multicentre trial. ${ }^{21}$ One moderate or severe migraine attack was treated in 668 patients. ${ }^{18}$ All treatments significantly improved response rates compared with placebo $(56.8,56.5,63.7$ and $42.4 \%$ for almotriptan 12.5, $25 \mathrm{mg}$, sumatriptan $100 \mathrm{mg}$ and placebo, respectively). Sumatriptan gave superior results for pain relief at 2 hours stratified by intensity of attack, but a higher percentage of patients were pain-free at 1 and 2 hours with either almotriptan dose. The rate of AEs was very low with all doses.

A further trial compared almotriptan $(12.5 \mathrm{mg}$ ) with sumatriptan (50 mg) in 1,173 patients with moderate or severe migraine.22 The findings showed that both treatments gave a similar rate of pain relief at 2 hours, but sumatriptan provided significantly better pain-free status $(p=0.05) .^{18}$ Migraine-associated symptoms and recurrence rates within 24 hours were similar with both treatments. No serious AEs were reported in any patient.

The sustained pain-free rate has been evaluated in a pooled analysis of three randomised, double-blind, placebo-controlled trials with almotriptan versus placebo or sumatriptan. ${ }^{23}$ Almotriptan treatment (6.25 and $12.5 \mathrm{mg}$ ) resulted in significantly better-sustained pain-free rates $(p<0.05$ versus placebo). Similar numbers of patients achieved sustained pain-free status in those treated with almotriptan or sumatriptan. ${ }^{23}$

Almotriptan is an effective and well-tolerated treatment for acute migraine and has been compared with rizatriptan in an open-label, crossover study in a real-world setting. ${ }^{24} \mathrm{~A}$ sub-study from a multicentre, open-label, crossover trial was conducted in 146 patients for the acute treatment of two migraine attacks using a sequential, crossover protocol. ${ }^{24}$ Significantly more patients taking rizatriptan had pain relief onset and more achieved pain freedom within 2 hours of dosing compared with those taking almotriptan ( 88.6 versus $73.4 \%, p=0.007$ and 55.7 versus $45.6 \%$, each $p=0.1$, respectively). In addition, in the rizatriptan group times to onset of pain freedom and pain relief were shorter than in the almotriptan group (median time 45 versus 60 minutes, $p=0.002$ and 100 versus 135 minutes, $p=0.004$ for pain relief and pain freedom, respectively). ${ }^{24}$ Of note, most patients preferred rizatriptan. ${ }^{24}$

\section{Eletriptan}

Eletriptan exhibits high affinity, selectivity and potent agonistic activity to human $5-\mathrm{HT}_{1 \mathrm{~B}}$ and $5-\mathrm{HT}_{1 \mathrm{D}}$ receptors. ${ }^{25}$ Affinity to the human $5-\mathrm{HT}_{1 \mathrm{~B}}$ and $5-\mathrm{HT}_{10}$ receptors is higher than observed with sumatriptan for the cranial artery compared with the coronary artery. ${ }^{25}$ Eletriptan is also rapidly absorbed and has good bioavailability following oral administration. ${ }^{25}$ Results from clinical trials indicate that eletriptan rapidly improves headache response and reduces headache recurrence. In addition, functional impairments are improved and the majority of AEs are mildto-moderate. ${ }^{25}$

Eletriptan (40 mg) has been shown to be one of the most effective acute migraine treatments in several meta-analyses. ${ }^{26}$ Furthermore, a post hoc analysis showed that patients who did not respond to the $40 \mathrm{mg}$ dose for three consecutive migraine attacks had a positive response to eletriptan $80 \mathrm{mg}$ versus placebo at 2 hours with the next three attacks ( $p<0.05$ for all comparisons); dose escalation may represent an effective strategy for around $50 \%$ of patients who show no response at a lower dose. ${ }^{26}$ 
Another analysis using results pooled from four randomised, doubleblind, placebo-controlled trials of eletriptan treatment for multi-attack migraine evaluated the effect of treating a second and third attack with the same $40 \mathrm{mg}$ dose in those who did not respond in the first attack to this treatment. ${ }^{26}$ In total 1,299 patients were combined from the studies with 297 who received eletriptan $40 \mathrm{mg}$ and 375 placebo, having no response to the first attack at 2 hours. Headache response at 2 hours for the first treated attack was significantly higher for treated patients compared with placebo (65.5 versus $28.7 \%, p<0.0001)$. Similar favourable results occurred with respect to pain-free at 2 hours, sustained headache response at24 hours and sustained pain-free at 24 hours for eletriptan versus placebo (all $p<0.0001$ ). In non-responding patients to the first attack, eletriptan treatment was significantly superior to placebo for second and third attacks on all efficacy measures (all $p<0.0001$ ) (Figure 1). These findings suggest that patients who do not respond to an initial dose of eletriptan may respond with the same dose to a second or third attack. ${ }^{26}$

Although eletriptan (80 mg) has shown significantly superior efficacy to sumatriptan (50 or $100 \mathrm{mg}$ ) in two clinical trials, this was only seen at a dose of $40 \mathrm{mg}$ in one trial. ${ }^{27}$ Therefore, eletriptan (40 mg) has been compared with sumatriptan $(100 \mathrm{mg}$ ) in a randomised, doubleblind, double-dummy, parallel-group trial $(n=2113)$ in single migraine attack to confirm the previous results. ${ }^{27}$ Eletriptan gave a significantly higher response rate at 2 hours than sumatriptan (67 versus 59\%, respectively, $\mathrm{p}<0.0001)$. For other endpoints, including 1 -hour headache response, 2-hour pain-free response, rescue medication use and sustained headache response, eletriptan was also superior $(p<0.05)$. Treatment-related AES were low with nausea the only one with $a \geq 2 \%$ incidence (eletriptan $4.9 \%$, sumatriptan $4.2 \%$ and placebo $2.8 \%$ ). This study therefore confirmed the better efficacy and tolerability with eletriptan than sumatriptan for treating migraine pain and restoring patient functioning. ${ }^{27}$

\section{Frovatriptan}

Frovatriptan has an extremely long half-life of approximately 25 hours, which is around five-times that of other triptans. ${ }^{28}$ Frovatriptan has high affinity for $5-\mathrm{HT}_{1 \mathrm{~B}}$ and $5-\mathrm{HT}_{1 \mathrm{D}}$ receptors and moderate affinity for $5-\mathrm{HT}_{1 \mathrm{~A}}$ and $5-\mathrm{HT}_{1 \mathrm{~F}} \mathrm{It}$ is one of the most potent $5-\mathrm{HT}_{1 \mathrm{~B}}$ agonists. ${ }^{28}$

Frovatriptan has distinctive pharmacokinetic and pharmacological properties, and was developed to offer a long duration of action and low likelihood of drug interactions and side effects. ${ }^{29-31}$ It is more selective for cerebral arteries compared with coronary arteries and is thus more suitable for treating those at risk from coronary artery disease. Although frovatriptan is mainly metabolised by cytochrome P450 (CYP) 1A2 it does not affect (inhibit or induce) this or other CYP isoenzymes. It also is not a substrate for monoamine oxidase-A in contrast to some other triptans and does not bind to plasma proteins to a large extent, hence it has a low risk of pharmacokinetic drug interactions. ${ }^{31}$

Results from three randomised, placebo-controlled, double-blind, parallel-group trials (2,676 migraine patients) indicated that headache response after 2 hours was significantly greater with frovatriptan compared with placebo $(p \leq 0.001)$ and was also approximately twice as effective at 4 hours. ${ }^{32}$ Furthermore, in most patients, time to headache response was within 1.5 hours and there was a low incidence (10-25\%) of 24-hour headache recurrence.

Frovatriptan has been evaluated in three double-blind, cross-over, headto-head trials versus rizatriptan, ${ }^{33}$ zolmitriptan ${ }^{34}$ and almotriptan..$^{35}$
Figure 1: Eletriptan response rate in second/third attack non-responding patients treated for a first attack ${ }^{26}$

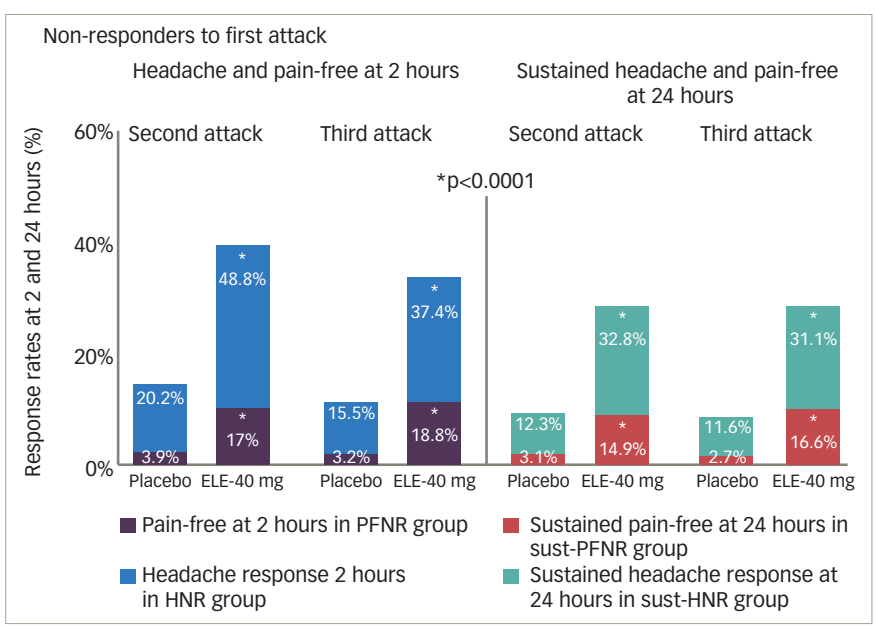

Sample size in second attack: headache response analysis (ELE, $n=170 ; P B O \quad n=193)$; pain-free analyses ( $E L E, n=341 ; P B O, n=254)$; sustained headache response analysis ( $E L E, n=235 ; P B O, n=227) ;$ Sustained pain-free analysis (ELE, $n=362 ; P B O, n=258$ ). Sample size in third attack: headache response analysis (ELE, $n=170 ; P B O, n=193$ ); pain-free analysis (ELE, $n=345 ; P B O, n=252) ;$ sustained headache response analysis ( $E L E, n=241 ; P B O, n=224)$ : sustained pain-free analysis ( $E L E, n=368 ; P B O, n=256)$. $E L E=$ electriptan $40 \mathrm{mg} ; H N R=$ headache non responders at 2 hours; $P B O=$ placebo; PFNR = pain-free non responders at 2 hours; sust-HNR = sustained headache non responders (24 hours); sust-PFNR = sustained pain-free non responders (24 hours). Reproduced with permission from Landy et al., 2014.26

The main aim of the study comparing frovatriptan and rizatriptan was to evaluate patient satisfaction for the treatment of acute migraine with either drug. ${ }^{33}$ Patients were recruited with a history of migraine and one or more attack in the previous 6 months. Treatment of 1-3 attacks for $<3$ months with either frovatriptan $(2.5 \mathrm{mg}$ ) or rizatriptan $(10 \mathrm{mg})$ was compared. The mean preference scores for 104 patients were similar for both treatments $(2.9 \pm 1.3$ versus $3.2 \pm 1.1$ for frovatriptan and rizatriptan, respectively). Furthermore, there was no significant difference for the secondary endpoints of rates of pain-free and pain-relief episodes at 2 hours or sustained pain-free episodes. However, frovatriptan resulted in significantly lower recurrent episodes than rizatriptan (21 versus $43 \%$ respectively, $p<0.001$ ) (Table 1). The number of patients with AEs was also similar. Thus, the efficacy of these two triptans is similar but frovatriptan has a greater duration of action..$^{33}$

Frovatriptan (2.5 mg) and zolmitriptan (2.5 mg) were compared in a study of 133 patients with a history of migraine using similar endpoints to the trial reported by Savi et al..$^{34}$ Both treatments were assigned similar preference scores (2.9 \pm 1.3 versus $3.0 \pm 1.3$ for frovatriptan and rizatriptan, respectively). No significant differences were noted for pain-free or pain-relief episodes at 2 hours, recurrence rate, or sustained pain-free, but recurrence time favoured frovatriptan in particular between hours 4-16 $(p<0.05)$. Significantly fewer treatment-related AEs occurred with frovatriptan than zolmitriptan $(p<0.05)$. In this study, frovatriptan offered the advantage of the two triptans with respect to recurrence and tolerability. ${ }^{34}$

The third study comparing frovatriptan (2.5 mg) and almotriptan (12.5 mg) had a very similar design with respect to patients enrolled and endpoints. ${ }^{35}$ In total, 114 patients completed the preference questionnaire. As with the other head-to-head studies there was no significant difference in patient preference, rates of pain-free ( 2 or 4 hours) and pain-relief ( 2 hours), sustained pain-free episodes and recurrent episodes were significantly less frequent with frovatriptan ( 30 versus $44 \%$ with almotriptan, $p<0.05$ ) and for recurrent episodes treated within 30 minutes $(p<0.05)$. Tolerability was also similar. ${ }^{35}$ 
Table 1: Frovatriptan versus rizatriptan for the acute treatment of migraine - secondary endpoints ${ }^{33}$

\begin{tabular}{|c|c|c|c|c|c|c|}
\hline & \multicolumn{2}{|c|}{$\begin{array}{l}\text { Intent to treat } \\
(n=125)\end{array}$} & \multirow[t]{2}{*}{$p$ value } & \multicolumn{2}{|c|}{$\begin{array}{l}\text { Per Protocol } \\
(n=96)\end{array}$} & \multirow[t]{2}{*}{$\mathrm{p}$ value } \\
\hline & $\begin{array}{l}\frac{}{\pi} \\
\frac{0}{2} \\
\frac{2}{2} \\
\frac{0}{0} \\
\frac{0}{10}\end{array}$ & 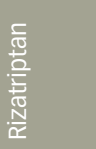 & & 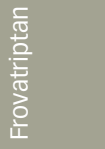 & $\begin{array}{l}\frac{}{\mathbb{d}} \\
\text { 을 } \\
\frac{1}{0} \\
\frac{N}{\alpha}\end{array}$ & \\
\hline $\begin{array}{l}\text { Pain-free episodes } \\
\text { at } 2 \mathrm{~h}\end{array}$ & 117 (33) & 141 (39) & NS & 107 (37) & $116(40)$ & NS \\
\hline $\begin{array}{l}\text { Recurrent episodes } \\
(\mathrm{IHS})\end{array}$ & $25(21)$ & $61(43)$ & $<0.001$ & $21(20)$ & 40 (35) & $<0.05$ \\
\hline $\begin{array}{l}\text { Recurrent episodes } \\
\text { (protocol) }\end{array}$ & 77 (22) & 117 (32) & $<0.001$ & $61(21)$ & 91 (32) & $<0.01$ \\
\hline $\begin{array}{l}\text { Sustained pain-free } \\
\text { episodes }\end{array}$ & $92(26)$ & $80(22)$ & NS & $86(30)$ & $76(26)$ & NS \\
\hline $\begin{array}{l}\text { Pain-relief episodes } \\
\text { at } 2 \mathrm{~h}\end{array}$ & $155(55)$ & $166(62)$ & NS & 129 (61) & $134(60)$ & NS \\
\hline
\end{tabular}

Data are reported as absolute and relative (\%) frequency. IHS = International Headache Society; NS = not significant. Reproduced with permission from Savi et al., 2011.33

The results from each of these three studies indicated that the triptans had similar efficacy in the immediate treatment of migraine, although frovatriptan was associated with lower recurrence rates, and thus improved sustained relief. However, in these studies, patients were instructed to take the drug as soon as the headache appeared (according to product labelling of all triptans). ${ }^{36-42}$ This could explain why no difference was seen between frovatriptan and other triptans at 2 hours. Moreover, the definition of recurrence used in these studies was from the International Headache Society Guidelines ${ }^{43}$ that is the more severe and affordable, and differs from many recurrence definitions used in a number of clinical trials of triptans.

\section{Naratriptan}

The efficacy of naratriptan $(2.5 \mathrm{mg}$ ) treatment for 1 year was assessed in an open-label study in acute migraine attacks $(n=417) .{ }^{44}$ Treated patients had headache relief in a median of $70 \%$ of moderate or severe and $86 \%$ of mild attacks, 4 hours after dosing. Furthermore, headache relief remained the same over prolonged treatment (0-6 versus 6-12 months) and frequency of naratriptan use (up to or over 36 attacks). AEs were absent in $84 \%$ of treated attacks and did not increase with respect to the number of doses. ${ }^{44}$

A comparative randomised, double-blind, crossover study comparing naratriptan with sumatriptan in 253 migraine patients (safety analysis) with a history of frequent headache recurrence has been reported. ${ }^{45}$ The efficacy analysis included 225 patients who treated two attacks. Headache recurrence occurred in $45 \%$ naratriptan-treated patients and $57 \%$ treated with sumatriptan 4-24 hours after treatment (not statistically significant). However, in patients with headache relief after two attacks 4-24 hours post-dosing, recurrence was more likely in the sumatriptan group ( 41 versus $57 \%$ respectively, $\mathrm{p}=0.005$ ). AEs were recorded in $22 \%$ of patients (naratriptan group) and 33\% for sumatriptan, and the incidence was similar following a second dose. In this study, these triptans gave similar efficacy, although in those who had headache relief after two attacks recurrence was significantly lower with naratriptan..$^{45}$

A meta-analysis of 10 RCTs (4,499 patients) with moderate or severe migraine attacks indicated that the rate ratio (RR) versus placebo for naratriptan $2.5 \mathrm{mg}$ was 2.52 (95\% confidence interval [CI] 1.78-3.57) and
Figure 2: Rizatriptan - total migraine freedom after treatment ${ }^{49}$

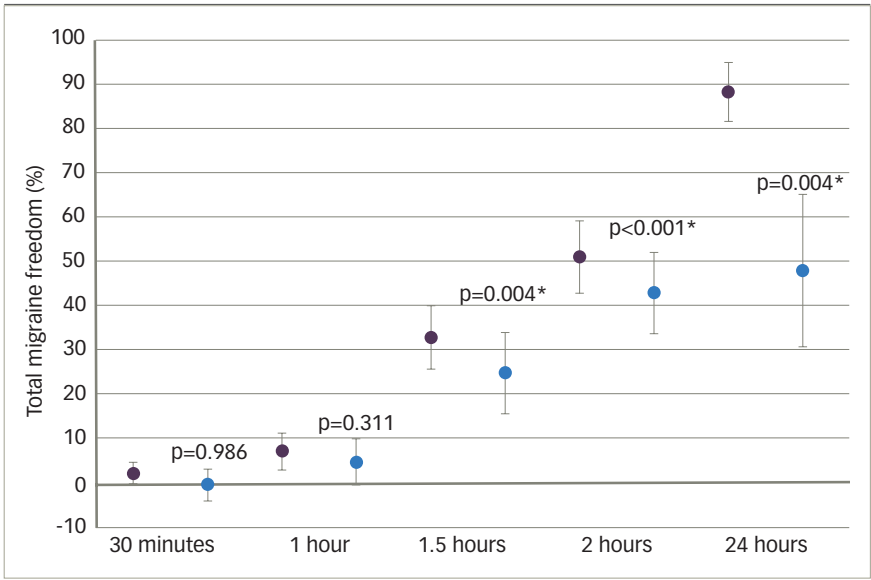

Total migraine freedom at various time points after oral rizatriptan $10 \mathrm{mg}$ (purple points) intake during an acute migraine attack. Blue points indicate the therapeutic gain (rizatriptan response-placebo response). Asterisks indicate a statistically significant $(p<0.05)$ therapeutic gain. Reproduced with permission from Barbanti et al., $2012^{49}$.

2.58 (1.99-3.35) for pain-free response at 2 and 4 hours. ${ }^{46}$ However, it was less effective in pain-free response than either rizatriptan or sumatriptan. In contrast, AEs were significantly fewer with naratriptan. ${ }^{46}$

Naratriptan has been evaluated as a preventative treatment of refractory chronic migraine compared with other treatments. ${ }^{47}$ In total, 27 patients who had failed on at least four preventative drugs were prescribed naratriptan twice daily $(2.5 \mathrm{mg})$. The frequency of headache days was significantly reduced by naratriptan at 2, 6 and 12 months $(p<0.001)$ and the number of days/month of severe pain significantly reduced at 1, 2, 6 and 12 months ( $p<0.01$ for all time points). The headache index was also reduced at 2,6 and 12 months (all $p<0.001$ ). ${ }^{47}$

\section{Rizatriptan}

Rizatriptan is rapidly absorbed, achieving plasma concentrations more quickly than other triptans. ${ }^{7}$ As with other triptans, rizatriptan causes contraction of isolated human coronary arteries in vitro - the effect occurs at high concentrations so myocardial ischaemia is unlikely to occur at plasma concentrations in patients with normal coronary circulation. It is available either as a tablet or as a wafer (orally disintegrating tablet [ODT]). ${ }^{48}$

The efficacy of rizatriptan has been demonstrated in randomised, double-blind, placebo-controlled trials. In one study, 80 patients with unilateral cranial autonomic symptoms were treated for a single moderate or severe migraine attack with $10 \mathrm{mg}$ rizatriptan (wafer) or placebo. ${ }^{49}$ Freedom from pain 2 hours after treatment occurred in 54 and $8 \%$ with rizatriptan versus placebo, respectively ( $46 \%$ therapeutic gain, $\mathrm{p}<0.001)$. In addition, significantly more rizatriptan-treated patients had total migraine freedom at 2 hours compared with placebo (51 and 8\% respectively; therapeutic gain $43 \%, p<0.001$ ) (Figure 2). ${ }^{49}$

Ferrari et al. conducted a meta-analysis of seven randomised, placebocontrolled, double-blind phase III trials of rizatriptan ( $10 \mathrm{mg}, \mathrm{n}=2,068$ or $5 \mathrm{mg}, \mathrm{n}=1,486)$ versus placebo $(n=1,260)$ for treating an acute migraine attack. $^{50}$ Rizatriptan (10 mg) was significantly better compared with placebo for pain relief at 2 hours (71 versus 38\%, $p<0.001$ ) and for the elimination of pain, nausea, photophobia, phonophobia and functional disability. In addition, more patients treated with rizatriptan than placebo had sustained pain relief over 24 hours (37 versus 18\% respectively, $\mathrm{p}<0.001)$. Except for the elimination of nausea, the $10 \mathrm{mg}$ dose was 
significantly more effective than $5 \mathrm{mg}$ on all endpoints at 2 hours and maintained over 24 hours (38 versus 32\% for rizatriptan $10 \mathrm{mg}$ and $5 \mathrm{mg}$, respectively, $\mathrm{p}=0.001) .{ }^{50}$

Rizatriptan (5 mg for body weight 20-39 kg, $10 \mathrm{mg}>40 \mathrm{~kg}$ ) has also been evaluated in 96 young patients aged 6-17 years in a double-blind, placebo-controlled, cross-over trial. ${ }^{51}$ Patients receiving either dose of rizatriptan achieved headache relief by two grades at 2 hours compared with placebo $(p<0.001)$. Rizatriptan was superior at 3 and 4 hours and was consistent over two treated attacks. Serious AEs were not evident. ${ }^{51}$

\section{Sumatriptan}

Sumatriptan is considered the gold standard triptan and is administered orally, subcutaneously (SC) or as a nasal spray. A Cochrane review considered the efficacy and tolerability of oral sumatriptan for treating a single acute migraine attack. ${ }^{52}$ Double-blind, RCTs were identified in which oral sumatriptan (100, 50 and $25 \mathrm{mg}$ ) was evaluated against placebo, no intervention, other drug treatments, behavioural therapy or physical therapy for an acute migraine attack in adults. In total, 25 RCTs with 16,200 patients were included. In the placebo-controlled trials, all doses of sumatriptan gave headache relief and relief of disability at 2 hours. With respect to pain-free response at 2 hours, numbers-neededto-treat (NNT) were 5.1 (3.9-7.1), and 7.5 (2.7-142) for the $100 \mathrm{mg}$ and $25 \mathrm{mg}$ dose, respectively. However, there was no significant difference between placebo and $50 \mathrm{mg}$. The NNT were similar with the three doses for headache relief. Sumatriptan was well tolerated, but AEs were more common with the 100-mg dose compared with placebo (risk difference [RD] 0.14). There were no significant differences for RDs between the 50 and $25 \mathrm{mg}$ doses and placebo. The authors concluded that oral sumatriptan is well tolerated and effective for treating a single acute migraine attack. Other triptans had similar effectiveness, but ergotamine plus caffeine was significantly less effective than sumatriptan. ${ }^{52}$

Many peripheral and central neural mechanisms contribute to the pathogenesis of migraine and have been identified as targets for acute and preventative treatment. Accordingly, treatment of migraine with a triptan and NSAID class, which have different mechanisms of action, can give better relief of migraine..$^{53}$ This strategy was evaluated in a multicentre, randomised, double-blind, double-dummy, placebocontrolled, four-arm study in 972 patients. A single moderate or severe migraine attack was treated with either naproxen sodium (500 mg), sumatriptan (50 mg), a combination of sumatriptan and naproxen (50 and $500 \mathrm{mg}$, respectively) or placebo. Sumatriptan plus naproxen was significantly more effective than sumatriptan or naproxen alone in achieving pain relief for 24 hours (46, 29 and 25 versus 17\% for the combination, sumatriptan alone, naproxen alone or the placebo group, respectively $[p<0.001])$. In addition, sumatriptan plus naproxen was significantly better than monotherapy in the 2-hour headache response, 2 -hour pain-free and sustained pain-free responses $(p<0.001)$ AEs were similar in all treatment groups. ${ }^{53}$

A total of 12 randomised, double-blind, placebo- or active-controlled studies of sumatriptan plus naproxen to treat an episode of migraine headache have been reviewed. ${ }^{54}$ Patients $(n=3,663)$ were treated for mild, moderate or severe pain attacks with sumatriptan ( 85 or $50 \mathrm{mg}$ ) plus naproxen $(500 \mathrm{mg}$ ). The findings showed that the combination was superior to placebo for pain-free and headache relief at 2 hours. The higher dose of sumatriptan did not significantly alter the findings, but early treatments were significantly more effective. Although AES were generally mild or moderate, they were more common with the combination compared with placebo. Thus, the combination treatment was more effective than the same dose of either drug alone..$^{54}$ Sumatriptan plus naproxen is now available in combination form. ${ }^{55}$

\section{Zolmitriptan}

An open-label phase II study of zolmitriptan permitted patients to choose treatment for initial, persistent or recurrent migraine headache. ${ }^{56} \mathrm{In}$ total, 49,784 patients were treated with 2.5 or $5 \mathrm{mg}$ zolmitriptan and $66 \%$ used a single dose. The 2-hour headache and pain-free response rate for all attacks was 85 and $79 \%$, and 69 and 59\%, respectively, for an initial dose of 2.5 or 5 mg zolmitriptan. In addition, both doses of zolmitriptan gave good headache $(84-91 \%$ and $76-84 \%$, respectively) and painfree response rates $(70-76 \%$ and $58-65 \%$, respectively) in patients treating at least 20 attacks. Furthermore, gender, age, presence of aura or relationship to menses had no effect on the treatment of multiple migraine attacks. ${ }^{56}$

Zolmitriptan has been assessed in a multicentre, randomised, doubleblind, placebo-controlled study in 2,122 migraine patients with or without aura. ${ }^{57}$ Zolmitriptan was administered as a $5 \mathrm{mg}$ nasal spray for the treatment of up to two migraine attacks within 15 minutes of pain being moderate or severe. Zolmitriptan treatment gave a significant headache response at 2 hours and at all earlier time points as early as 15 minutes post-dose $(66.2 \%$ of treated patients compared with $35 \%$ on placebo, $p<0.001$ for headache response at 2 hours and $p<0.001$ for earlier time points). Zolmitriptan treatment was also better than placebo based on pain-free rates, and headache response at 4 hours, and sustained headache response and pain-free response at 24 hours. AEs were mild or moderate and of short duration with zolmitriptan. These results indicate that zolmitriptan is highly effective in treating acute migraine with very fast onset of action (15 minutes post-dose), a sustained response and good tolerability. ${ }^{57}$

Zolmitriptan has also demonstrated efficacy and good tolerability for the treatment of menstrual migraine attacks in a multicentre, randomised, double-blind, placebo-controlled, parallel-group outpatient study..$^{58}$

The Zomig treatment of acute migraine headache in adolescents (TEENZ) study assessed the efficacy of zolmitriptan nasal spray versus placebo for the acute treatment of a single episode of adolescent migraine in patients $12-17$ years of age. ${ }^{59}$ In this randomised, doubleblind, placebo-controlled trial 789 patients were treated with either $5,2.5$, or $0.5 \mathrm{mg}$ zolmitriptan nasal spray or placebo. An interim analysis showed that doses of 0.5 and $2.5 \mathrm{mg}$ were ineffective compared with placebo, but zolmitriptan $5.0 \mathrm{mg}$ was significantly more effective than placebo ( $p<0.001)$. Zolmitriptan $5.0 \mathrm{mg}$ was also significantly better than placebo with respect to pain-free status 3 or 4 hours posttreatment and in achieving headache response after 2, 3 and 4 hours $(p<0.001$ and $p \leq 0.011)$. All doses of zomitriptan were well tolerated with dysgeusia the most frequent $A E$ and no serious AEs or AEs leading to discontinuation. Furthermore, the majority of AEs were mild or moderate and consistent with the known profile of zomitriptan in adult and adolescents. ${ }^{59}$

\section{Serotonin syndrome}

When triptans and selective serotonin reuptake inhibitors (SSRIS) or serotonin-noradrenaline reuptake inhibitors (SNRIS) are prescribed together, there is a risk of potentially fatal serotonin syndrome..$^{60} \mathrm{~A}$ warning is triggered with most drug interaction programmes when these drugs are co-prescribed. In 2006 the US Food and Drug Administration issued a warning for this risk, but there is a lack of consensus as the number of cases of serotonin syndrome are extremely low, the interaction is 
biologically implausible and triptans are used relatively infrequently. The American Headache Society concluded that there are inadequate data to assess the risk and evidence does not support limiting the use of the combination, but caution is needed when a triptan and SSRI or SNRI are administered. ${ }^{.1}$ Generally, the AES associated with triptans occur infrequently and are benign. Central nervous system AEs have been reported in up to $15 \%$ of patients, but vary among triptans with almotriptan, naratriptan and sumatriptan having the lowest incidence. ${ }^{62}$

Triptans are contraindicated with a number of conditions including cardiovascular disease, during pregnancy and breastfeeding, in combination with monoamine oxidase inhibitors or ergot compounds, and for familial hemiplegic migraine and basilar migraine. ${ }^{36-41,63-65}$

\section{Practical considerations when selecting a triptan for the treatment of migraine}

Triptans are the first-line option to immediately treat acute moderateto-severe migraines. Each triptan has different pharmacokinetic and pharmacodynamic properties and formulation. Some have several formulations such as tablets, dissolvable tablets, nasal sprays and injections. Many studies have been completed with triptans but they have not all been tested head-to-head in a single study. In order to select the optimum triptan for an individual patient, efficacy, safety and both pharmacokinetic and pharmacodynamic properties should be considered. In addition, the clinical characteristics of the migraine attack and the lifestyle of the patient and medical history are important. ${ }^{64}$

The properties of triptans can guide the optimum drug to prescribe for different migraine attacks. For example, where there is a high rate of recurrence, a long-, fast-acting triptan is most suitable to achieve rapid peak intensity or nocturnal onset, and triptans administered by SC injection, nasal spray, or suppository for early onset, or where there is a preponderance of vegetative symptoms. ${ }^{64}$

Meta-analyses of triptan treatment have been published. Cameron and colleagues conducted a systemic review and network meta-analysis comparing the relative efficacy of triptans using the Cochrane Library, Medline and Embase (133 randomised trials). ${ }^{14}$ The main efficacy variables (headache relief within 2 hours, 2-hour sustained freedom from pain, sustained headache relief and freedom from pain at 24 hours) were met by standard doses of triptans with rates from 18-76\% of patients. Sumatriptan (SC), rizatriptan (ODT), zolmitriptan (ODT) and eletriptan tablets were associated with the best outcomes. ${ }^{14}$

A similar network meta-analysis comparing NSAIDs and triptans concluded that eletriptan was probably the most suitable drug, although the excellent tolerability of ibuprofen makes that a good choice too. combination therapy may also be a promising future approach. ${ }^{66}$

Thorlund et al. conducted a Bayesian multiple treatment comparison meta-analysis of seven triptans used in adults to abort migraine attacks from 74 double-blind, randomised clinical trials. ${ }^{67}$ Although all triptans were significantly superior to placebo (odds ratio [OR], 95\% credible interval precluding 1.00), eletriptan produced the most favourable outcome in producing sustained pain-free responses. ${ }^{67}$

All triptans are effective compared with placebo at marketed doses with good tolerability. Treatment-related AEs are generally mild and transient i.e., quickly disappear.

\section{Conclusions}

All of the currently licensed triptans are effective in a number of manifestations of migraine. They have a number of different formulations which have to be considered when a triptan is prescribed and it is important that the most effective one is selected for each patient. Studies have shown that the formulations do not have the same efficacy in every indication, for example zolmitriptan nasal spray. In addition, published meta-analyses of studies have provided information to assist prescribers to choose the best option. Generally, AEs are mild to moderate with all the triptans. However, there is currently no consensus as to the role of triptans with respect to serotonin syndrome, but doctors need to be aware of the possible problems when triptans and SSRIS or SNRIs are coprescribed. Triptans are contraindicated with several diseases especially those of the cardiovascular system.

The third edition of the International Headache Society guidelines for controlled trials of drugs in migraine includes recommendations for the incidence of a recurrence. ${ }^{68}$ After 2-hour pain freedom, any headache pain from 2-48 hours following study drug administration, regardless of its severity, should be considered a recurrence. Previously recurrence or relapse has been defined as occurring when a study participant gains pain relief initially (improvement for moderate or severe pain at baseline to mild or no pain at the primary efficacy time point) and subsequently experiences a moderate or severe headache from the time point of primary efficacy and up to 24 hours. The main drawback associated with triptans is headache recurrence and the underlying mechanisms are not well understood. ${ }^{69}$ Rates of recurrence vary with each triptan. Geraud et al. investigated mean recurrence rates from the results of 31 triptan efficacy studies. ${ }^{69}$ Frovatriptan gave the lowest rate with rizatriptan the highest (17 and $40 \%$, respectively), with elimination half-life inversely correlated with recurrence $(p=0.0016)$. Furthermore, $5-\mathrm{HT}_{1 \mathrm{~B}}$ but not 5- $\mathrm{HT}_{1 \mathrm{D}}$ receptor potency was significantly correlated with recurrence ( $p=0.034$ and 0.54 , respectively). Migraine recurrence is affected by the pharmacological and pharmacokinetic properties of the triptan but not related to initial clinical efficacy. The triptans with a longer half-life and largest $5-\mathrm{HT}_{1 \mathrm{~B}}$ receptor affinity have the lowest rates of headache recurrence. ${ }^{69}$

Triptans are effective treatments for acute migraine, but have different properties in terms of efficacy, tolerability and pharmacokinetics and pharmacodynamics. These attributes need to be taken into consideration when selecting the optimum triptan for treating a patient with acute migraine. Furthermore, combination therapy with a triptan and another drug should be explored as this approach may enhance their effectiveness. $]$
Ahmed F, Parthasarathy R, Khalil M, Chronic daily headaches, Ann Indian Acad Neurol, 2012;15:S40-50.

2. Murray CJ, Barber RM, Foreman KJ, et al., Global, regional, and national disability-adjusted life-years (DALYS) for 315 diseases and injuries and healthy life expectancy (HALE), 1990-2015: a systematic analysis for the Global Burden of Disease Study 2015 Lancet, 2016;388:1603-58.

3. Steiner TJ, Stovner $\sqcup$, Vos T, GBD 2015: migraine is the third cause of disability in under 50s, J Headache Pain, 2016;17:104. Dahlof $\mathrm{C}$, Linde $\mathrm{M}$, One-year prevalence of migraine in
Sweden: a population-based study in adults, Cephalalgia 2001;21:664-71.

5. Wang SJ, Fuh JL, Young YH, et al., Prevalence of migraine in Taipei, Taiwan: a population-based survey, Cephalalgia, 2000;20:566-72.

6. Strine TW, Chapman DP, Balluz LS, Population-based US study of severe headaches in adults: psychological distress and comorbidities, Headache, 2006;46:223-32.

7. Jette N, Patten S, Williams J, et al., Comorbidity of migraine and psychiatric disorders - a national population-based study
Headache, 2008:48:501-16.

8. Fernandez-de-Las-Penas C, Hernandez-Barrera V, CarrascoGarrido P, et al., Population-based study of migraine in Spanish adults: relation to socio-demographic factors, lifestyle and co-morbidity with other conditions, J Headache Pain, 2010;11:97-104.

9. Jensen R, Stovner $L$, Epidemiology and comorbidity of headache, Lancet Neurol, 2008;7:354-61.

10. Stovner $\downarrow$, Andree $C$, Prevalence of headache in Europe: a review for the Eurolight project, $J$ Headache Pain, 
2010:11:289-99.

11. Migraine Research Foundation. Migraine in women. Available at: https://migraineresearchfoundation.org/about-migraine/ migraine-in-women/ (accessed 23 February 2017)

12. National Institute for Health and Care Evidence (NICE), Headaches in over 12s: diagnosis and management. Clinical guideline [CG150], 2015. Available at: www.nice.org.uk/ guidance/cg150 (accessed 23 February 2017).

13. Silberstein SD, Holland S, Freitag F, et al., Evidence-based guideline update: pharmacologic treatment for episodic migraine prevention in adults. Report of the Quality Standards Subcommittee of the American Academy of Neurology and the American Headache Society, Neurology, 2012;78:1337-45.

14. Cameron C, Kelly S, Hsieh SC, et al., Triptans in the acute treatment of migraine: a systematic review and network metaanalysis, Headache, 2015;55 Suppl. 4:221-35.

15. Ahn $\mathrm{AH}$, Basbaum Al, Where do triptans act in the treatment of migraine?, Pain, 2005; 115:1-4

16. MCMahon G Triptans for migraine: how should triptans be used?, 2010. Available at: http://blogs.nejm.org/now/index.php/ triptans-for-migraine-how-should-triptans-be-used/2010/07/02/ (accessed 23 February 2017).

17. Capi M, Curto M, Lionetto L, et al., Eletriptan in the management of acute migraine: an update on the evidence for efficacy, safety, and consistent response, Ther Adv Neurol Disord, 2016;9:414-23.

18. Sandrini G, Perrotta A, Arce Leal NL, et al., Almotriptan in the treatment of migraine, Neuropsychiatr Dis Treat, 2007:3:799-809.

19. Pascual J Falk RM, Piessens F, et al, Consistent efficacy and tolerability of almotriptan in the acute treatment of multiple migraine attacks: results of a large, randomized, double-blind, placebo-controlled study, Cephalalgia, 2000;20:588-96.

20. Dahlof C, Tfelt-Hansen P, Massiou H, et al., Dose finding, placebo-controlled study of oral almotriptan in the acute placebo-controlled study of oral almotriptan in the acut

21. Dowson AJ, Massiou H, Lainez JM, et al., Almotriptan is an effective and well-tolerated treatment for migraine pain: results of a randomized, double-blind, placebo-controlled clinical trial, Cephalalgia, 2002;22:453-61.

22. Spierings EL, Gomez-Mancilla B, Grosz DE, et al., Oral almotriptan vs. oral sumatriptan in the abortive treatment of migraine: a double-blind, randomized, parallel-group, optimumdose comparison, Arch Neurol, 2001;58:944-50.

23. Dodick DW, Almotriptan increases sustained pain-free outcomes in acute migraine: results from three controlled clinical trials, Headache, 2002:42:21-7.

24. Ng-Mak DS, Hu XH, Bigal M, Migraine treatment with rizatriptan and almotriptan: a crossover study, Headache, 2009:49:655-62. amote M, Pharmacological, pharmacokinetic and clinical profile of eletriptan (Relpax), a new triptan for migraine, Nihon Yakurigaku Zasshi, 2003;122:93-101.

26. Landy SH, Tepper SJ, Schweizer E, et al., Outcome for headache and pain-free nonresponders to treatment of the first attack: a pooled post-hoc analysis of four randomized trials of eletriptan 40 mg, Cephalalgia, 2014;34:376-81.

27. Mathew NT, Schoenen J, Winner P, et al., Comparative efficacy of eletriptan $40 \mathrm{mg}$ versus sumatriptan $100 \mathrm{mg}$, Headache, 2003;43:214-22.

28. Kelman $L$, Review of frovatriptan in the treatment of migraine Neuropsychiatr Dis Treat, 2008:4:49-54.

29. Tfelt-Hansen P, De Vries P, Saxena PR, Triptans in migraine: a comparative review of pharmacology, pharmacokinetics and efficacy, Drugs, 2000;60:1259-87.
30. Balbisi EA, Frovatriptan succinate, a 5-HT1B/1D receptor agonist for migraine, Int J Clin Pract, 2004:58:695-705

31. Buchan $P$, Wade $A$, Ward $C$, et al., Frovatriptan: a review of drugdrug interactions, Headache, 2002;42 Suppl. 2:S63-73.

32. Ryan R, Geraud G, Goldstein J, et al., Clinical efficacy of frovatriptan: placebo-controlled studies, Headache, 2002;42 Suppl. 2:S84-92.

33. Savi L, Omboni S, Lisotto C, et al., A double-blind, randomized, multicenter, Italian study of frovatriptan versus rizatriptan for the acute treatment of migraine, $J$ Headache Pain 2011;12:219-26.

34. Tullo V, Allais G, Ferrari MD, et al., Frovatriptan versus zolmitriptan for the acute treatment of migraine: a doubleblind, randomized, multicenter, Italian study, Neurol Sci, 2010;31 Suppl. 1:S51-4.

35. Bartolini M, Giamberardino MA, Lisotto C, et al., A double-blind, randomized, multicenter, Italian study of frovatriptan versus almotriptan for the acute treatment of migraine, $J$ Headache Pain, 2011:12:361-8

36. Sumatriptan $50 \mathrm{mg}$ tablets SPC, 2015. Available at: www. medicines.org.uk/emc/medicine/23027 (accessed 23 February 2017).

37. Naratriptan $2.5 \mathrm{mg}$ film-coated tablets SPC, 2012. Available at: www.medicines.org.uk/emc/medicine/26134/SPC/ Naratriptan+2.5mg+Film-coated+Tablets (accessed 23 February 2017)

38. Almogran $12.5 \mathrm{mg}$ film-coated tablets SPC, 2015. Available at: uww.medicines.org.uk/emc/medicine/21973 (accessed 23 February 2017).

39. Migard $2.5 \mathrm{mg}$ film-coated tablets SPC, 2017. Available at: www.medicines.org.uk/emc/medicine/15216 (accessed 23 February 2017).

40. Rizatriptan $10 \mathrm{mg}$ tablets SPC, 2015. Available at: www medicines.org.uk/emc/medicine/31041 (accessed 23 February 2017)

41. Zomig tablets $2.5 \mathrm{mg}$ SPC, 2017. Available at: www.medicines org.uk/emc/medicine/5905 (accessed 23 February 2017).

42. Relpax $20 \mathrm{mg}$ film-coated tablets SPC, 2014. Available at: www.medicines.org.uk/emc/medicine/8195 (accessed 23 February 2017).

43. International Headache Society (IHS) Classification ICHD-II. Available at: www.ihs-klassifikation.de/en/02_klassifikation/02 teil1/01.02.00_migraine.html (accessed 23 February 2017).

44. Heywood J, Bomhof MA Pradalier A et al on behalf of the Naratriptan Long-term Study Group. Tolerability and efficacy of naratriptan tablets in the acute treatment of migraine attacks naratriptan tablets in the acute treat

45. Gobel $H$, Winter $P$ Boswell $D$, et al, Comparison of naratriptan and sumatriptan in recurrence-prone migraine patients. Naratriptan International Recurrence Study Group, Clin Ther, 2000;22:981-9

46. Ashcroft DM, Millson D, Naratriptan for the treatment of acute migraine: meta-analysis of randomised controlled trials, Pharmacoepidemiol Drug Saf, 2004;13:73-82.

47. Rapoport AM, Bigal ME, Volcy M, et al., Naratriptan in the preventive treatment of refractory chronic migraine: a review of 27 cases, Headache, 2003;43:482-9.

48. Lainez MJ, Rizatriptan in the treatment of migraine, Neuropsychiatr Dis Treat, 2006;2:247-59.

49. Barbanti P, Fofi L, Dall'Armi V, et al., Rizatriptan in migraineurs with unilateral cranial autonomic symptoms: a double-blind trial, J Headache Pain, 2012:13:407-14.
50. Ferrari MD, Loder E, MCCarroll KA, et al., Meta-analysis of rizatriptan efficacy in randomized controlled clinical trials, Cephalalgia, 2001:21:129-36.

51. Ahonen $\mathrm{K}$, Hamalainen ML, Eerola M, et al., A randomized trial of rizatriptan in migraine attacks in children, Neurology, 2006;67:1135-40.

52. McCrory DC, Gray RN, Oral sumatriptan for acute migraine, Cochrane Database Syst Rev, 2003:CD002915.

53. Smith TR, Sunshine A, Stark SR, et al., Sumatriptan and naproxen sodium for the acute treatment of migraine, Headache, 2005;45:983-91.

54. Law S, Derry S, Moore RA, Sumatriptan plus naproxen for the treatment of acute migraine attacks in adults, cochrane Database Syst Rev, 2016:CD008541.

55. Treximet tablets, prescribing information, 2011. Available at: www.accessdata.fda.gov/drugsatfda_docs/ label/2011/021926s002lbl.pdf (accessed 23 February 2017).

56. Tuchman M, Edvinsson L, Geraud G, et al., Zolmitriptan provides consistent migraine relief when used in the long-term, Curr Med Res Opin, 1999;15:272-81.

57. Dodick D, Brandes J, Elkind A, et al., Speed of onset, efficacy and tolerability of zolmitriptan nasal spray in the acute treatment of migraine: a randomised, double-blind, placebocontrolled study, CNS Drugs, 2005;19:125-36.

58. Tuchman M, Hee A, Emeribe U, et al., Efficacy and tolerability of zolmitriptan oral tablet in the acute treatment of menstrua migraine, CNS Drugs, 2006;20:1019-26.

59. Winner P, Farkas V, Stillova $\mathrm{H}$, et al., Efficacy and tolerability of zolmitriptan nasal spray for the treatment of acute migraine in adolescents: results of a randomized, doubleblind, multi-center, parallel-group study (TEENZ), Headache 2016:56:1107-19.

60. Gören JL, Wiseman CN, Can combining triptans with SSRIS or SNRIs cause serotonin syndrome? Current Psychiatry, 2012;11:E1-E2.

61. Evans RW, Tepper SJ, Shapiro RE, et al., The FDA alert on serotonin syndrome with use of triptans combined with selective serotonin reuptake inhibitors or selective serotoninnorepinephrine reuptake inhibitors: American Headache Society position paper, Headache, 2010;50:1089-99.

62. Dodick DW, Martin V, Triptans and CNS side-effects: pharmacokinetic and metabolic mechanisms, Cephalalgia, 2004;24:417-24

63. Huffman GB, Evaluating and treating unintentional weight loss in the elderly, Am Fam Physician, 2002;65:640-50

64. Belvis R, Pagonabarraga J, Kulisevsky J, Individual triptan selection in migraine attack therapy, Recent Pat CNS Drug Discov, 2009:4:70-81.

65. Jamieson DG, The safety of triptans in the treatment of patients with migraine, Am J Med, 2002:112:135-40

66. Xu H, Han W, Wang J, et al., Network meta-analysis of migraine disorder treatment by NSAIDs and triptans, J Headache Pain 2016;17:113.

67. Thorlund K, Mills EJ, Wu P, et al., Comparative efficacy of triptans for the abortive treatment of migraine: a multiple treatment comparison meta-analysis, Cephalalgia, 2014;34:258-67.

68. Tfelt-Hansen P, Pascual J, Ramadan N, et al., Guidelines for controlled trials of drugs in migraine: third edition. A guide for investigators, Cephalalgia, 2012;32:6-38.

69. Geraud G, Keywood C, Senard JM, Migraine headache recurrence: relationship to clinical, pharmacological, and pharmacokinetic properties of triptans, Headache, 2003:43:376-88. 